\title{
Surgical Management of Vulvar Cancer
}

\author{
Thanh H. Dellinger, MDa; Amy A. Hakim, MDa; Stephen J. Lee, MDª Mark T. Wakabayashi, MDa; \\ Robert J. Morgan, $\mathrm{MD}^{\mathrm{b}}$; and Ernest S. Han, MD
}

\begin{abstract}
Vulvar cancer is a rare malignancy with high curability in early-stage disease, yet poor outcomes for advanced-stage and recurrent disease. Surgical management is at the cornerstone of treatment for most vulvar cancers, and includes conservative and radical resection of the primary vulvar tumor and excision of local lymph nodes, which are major prognostic factors and drive adjuvant treatment. This review summarizes the surgical management of primary squamous cell carcinoma of the vulva, specifically initial treatment guidelines by stage, based on the 2017 NCCN Clinical Practice Guidelines in Oncology for Vulvar Cancer.
\end{abstract}

J Natl Compr Canc Netw 2017;15(1):121-128

\section{NCCN: Continuing Education}

\section{Accreditation Statement}

This activity is designed to meet the educational needs of physicians and nurses involved in the management of patients with cancer. No commercial support was received for this article. Fees: There are no fees associated with participation of this activity.

National Comprehensive Cancer Network is accredited by the Accreditation Council for Continuing Medical Education (ACCME) to provide continuing medical education for physicians.

NCCN designates this journal-based CE activity for a maximum of 1.0 AMA PRA Category 1 Credit ${ }^{\mathrm{TM}}$. Physicians should claim only the credit commensurate with the extent of their participation in the activity.

NCCN is accredited as a provider of continuing nursing education by the American Nurses Credentialing Center's Commission on Accreditation.
NCCN designates this educational activity for a maximum of 1.0 contact hour.

All clinicians completing this activity will be issued a certificate of participation. To participate in this journal CE activity: 1) review the educational content; 2 ) take the posttest with a $66 \%$ minimum passing score and complete the evaluation at http://education.nccn.org/ node/80141; and 3) view/print certificate.

Release date: January 3, 2017; Expiration date: January 3, 2018

\section{Learning Objectives}

Upon completion of this activity, participants will be able to:

- Summarize the recommended treatment approaches for vulvar cancer by stage

- Discuss options for the surgical evaluation and treatment of vulvar cancer
From a Department of Surgery, Division of Gynecologic Oncology, and ${ }^{b}$ Department of Medical Oncology, City of Hope National Medical Center, Duarte, California.

Submitted October 3, 2016; accepted for publication November 28, 2016. The authors have disclosed that they have no financial interests, arrangements, affiliations, or commercial interests with the manufacturers of any products discussed in this article or their competitors.

Correspondence: Thanh H. Dellinger, MD, Department of Surgery, Division of Gynecologic Oncology, City of Hope Comprehensive Cancer Center, 1500 East Duarte Road, Duarte, CA 91010. E-mail: tdellinger@coh.org

\section{EDITOR}

Kerrin M. Green, MA, Assistant Managing Editor, Journal of the National Comprehensive Cancer Network, has disclosed that she has no relevant financial relationships.

\section{JNCCN}

Kimberly Callan, MS, Senior Director, Professional and Patient Publications, disclosed that she has no relevant financial relationships. Genevieve Emberger Hartzman, MA, Journal Production Specialist, has disclosed that she has no relevant financial relationships.

\section{CE PLANNERS:}

Deborah J. Moonan, RN, BSN, Director, Continuing Education, has disclosed that she has no relevant financial relationships.

Karen Kanefield, Manager, Continuing Education Accreditation and Program Operations, has disclosed that she has no relevant financial relationships.

Kristina M. Gregory, RN, MSN, OCN, Vice President, Clinical Information Operations, has disclosed that she has no relevant financial relationships. Rashmi Kumar, PhD, Senior Manager, Clinical Content, has disclosed that she has no relevant financial relationships

Jillian L. Scavone, PhD, Oncology Scientist/Medical Writer, has disclosed that she has no relevant financial relationships. 


\section{Background}

Vulvar cancer affects approximately 5,100 women a year in the United States, resulting in approximately 1,000 deaths per year. ${ }^{1}$ The most common histology is squamous cell carcinoma of the vulva, or vulvar cancer. The pathogenesis of this disease arises from either human papillomavirus (HPV)-related dysplasia, affecting primarily younger women, or from vulvar dermatoses, such as lichen sclerosis, frequently affecting older patients. HPV-related vulvar dysplasia includes vulvar intraepithelial neoplasia (VIN) I, II, and III; the rate of progression from VIN III to invasive cancer is $23 \% .^{2}$ Risk factors associated with HPV-related vulvar cancers include smoking, sexually transmitted diseases, and immunosuppression, whereas in older patients with dermatosis-related vulvar cancer, obesity, diabetes mellitus, and hypertension are frequently present. The clinical presentation in most patients includes vulvar pruritus or a self-palpated lesion, although frequently a vulvar lesion is detected by a healthcare provider. Late-presenting symptoms include bleeding, pain, vaginal discharge, and difficulty with urination or defecation.

\section{Staging}

Vulvar cancer is staged surgically, based on the 2009 FIGO and AJCC TNM staging (Table 1). Staging relies on a vulvar biopsy to determine stromal invasion, followed by clinical assessment of tumor size and clinical, surgical, and/or radiologic assessment of groin nodes and distant metastases, including pelvic lymph node spread.

\section{Diagnosis and Workup}

Diagnosis relies on a liberal biopsy of the vulvar lesion at the peripheral edge of the tumor, to include the underlying stroma and avoid the necrotic center of the tumor. This can be accomplished in the office with either a Tischler or a Keyes punch biopsy instrument. All suspicious vulvar lesions should be biopsied. Further examination should focus on the size and location of the primary vulvar tumor in relation to vital adjacent organs (urethra, clitoris, anus, rectum), its relation to the midline, and presence of multifocal disease. Initial workup includes several imaging studies depending on clinical presentation. ${ }^{3}$
Consideration should be given initially to a plain chest radiograph, followed by a chest CT without contrast if an abnormality is noted. For early-stage disease, a pelvic MRI may further define the vulvar tumor and locoregional disease spread, whereas for suspected metastatic or advanced-stage disease, a CT scan of the chest, abdomen, and pelvis or a wholebody PET/CT scan should be considered to evaluate for pelvic nodal and distant spread. ${ }^{4}$

The status of the groin lymph nodes drives adjuvant treatment, and a groin ultrasound may be considered to assess for adenopathy in select patients. In general, palpation and assessment of clinically suspicious groin nodes leads to the decision to proceed with either surgical (inguinofemoral lymphadenectomy [IFLND]) or image-guided biopsy to confirm the groin node status. Notably, clinical palpation of groin nodes carries a $24 \%$ false-negative rate based on a national cooperative group study finding in $1987,{ }^{5}$ thereby emphasizing the need for surgical staging in clinically negative groin nodes. Table 2 shows the clinicopathologic factors predicting inguinal lymph node metastases based on a Gynecologic Oncology Group study (GOG Protocol 36) of 588 vulvar cancer cases. ${ }^{5}$

\section{Treatment}

\section{Overview}

The treatment of stage I disease confined to the vulva is primary surgical resection, with adjuvant radiation (RT) reserved for those with high-risk factors. Locally advanced disease (stage II-IVA) is treated with a combination of radical surgery and adjuvant chemoradiation. Occasionally, neoadjuvant chemoradiation may be used to reduce tumor burden in these patients in order to facilitate surgical resection and potentially avoid a pelvic exenteration. For stage IVB vulvar cancer with distant metastases, palliation with chemotherapy and/or RT is recommended. Treatment guidelines by stage are outlined in Table 1, based on NCCN Clinical Practice Guidelines in Oncology (NCCN Guidelines) for Vulvar Cancer, and further described hererin. ${ }^{3}$

\section{Early-Stage Vulvar Cancer (Stage I and II, $\leq \mathbf{4} \mathrm{cm}$ )}

Stage I vulvar cancer is defined as tumor confined to the vulva without nodal spread (T1NO). The important prognostic clinicopathologic criteria are stromal invasion $(1 \mathrm{~mm})$ and tumor size $(4 \mathrm{~cm})$. For patients 
Vulvar Cancer Treatment

\begin{tabular}{|c|c|c|c|c|c|c|c|}
\hline TNM & Stage & Substage & Pathology & \multicolumn{2}{|l|}{ Treatment } & \multicolumn{2}{|c|}{ Adjuvant Treatment $\quad$ Follow-Up } \\
\hline \multirow[t]{6}{*}{ T1 } & \multirow{6}{*}{$\begin{array}{l}\text { Tumor } \\
\text { confined } \\
\text { to vulva }\end{array}$} & \multirow[t]{2}{*}{$\mathrm{IA}(\leq 2 \mathrm{~cm})$} & $\mathrm{IA} \leq 1 \mathrm{~mm}$ & \multicolumn{2}{|l|}{ Wide local excision } & \multicolumn{2}{|c|}{ Modified radical vulvectomy if $>1 \mathrm{~mm}$ invasion } \\
\hline & & & $\mathrm{IA}>1 \mathrm{~mm}$ & \multirow[t]{2}{*}{$\begin{array}{l}\text { Modified radical } \\
\text { vulvectomy + groin } \\
\text { LN dissection }\end{array}$} & Negative margins & \multicolumn{2}{|c|}{$\begin{array}{l}\text { Consider radiation if risk factors present: } \\
\text { - Lymphovascular space invasion } \\
\text { - Close margins }(<8 \mathrm{~mm}) \\
\text { - Large tumor size } \\
\text { - Deep invasion } \\
\text { - Spray or diffuse pattern of disease }\end{array}$} \\
\hline & & \multirow[t]{4}{*}{$\mathrm{IB}(>2 \mathrm{~cm})$} & $\mathrm{IB} \leq 4 \mathrm{~cm}$ & & Positive margins & \multicolumn{2}{|c|}{ Reexcision or radiation } \\
\hline & & & $\mathrm{IB}>4 \mathrm{~cm}$ & Clinically (-) LN $\rightarrow$ & IFLND: (-) LN & \multicolumn{2}{|l|}{ Chemoradiation to tumor } \\
\hline & & & & & IFLND: (+) LN & \multirow{2}{*}{\multicolumn{2}{|c|}{ Chemoradiation to tumor, groins, pelvis }} \\
\hline & & & & Clinically (+) LN $\rightarrow$ & Biopsy-proven & & \\
\hline \multirow[t]{4}{*}{$\mathrm{T} 2$} & \multirow[t]{4}{*}{$\begin{array}{l}\text { II } \\
\text { Lower } \\
\text { one-third } \\
\text { of urethra, } \\
\text { vagina, or } \\
\text { anus }\end{array}$} & \multirow{2}{*}{\multicolumn{2}{|c|}{$\mathrm{II} \leq 4 \mathrm{~cm}$}} & \multirow[t]{2}{*}{$\begin{array}{l}\text { Modified radical } \\
\text { vulvectomy + groin } \\
\text { LN }^{\mathrm{a}}\end{array}$} & Negative margins & \multicolumn{2}{|c|}{$\begin{array}{l}\text { Consider radiation if risk factors present: } \\
\text { - Lymphovascular space invasion } \\
\text { - Close margins }(<8 \mathrm{~mm}) \\
\text { - Large tumor size } \\
\text { - Deep invasion } \\
\text { - Spray or diffuse pattern of disease }\end{array}$} \\
\hline & & & & & Positive margins & \multicolumn{2}{|c|}{ Reexcision or radiation } \\
\hline & & \multirow{2}{*}{\multicolumn{2}{|c|}{ II $>4 \mathrm{~cm}$}} & \multirow[t]{2}{*}{ Clinically (-) LN $\rightarrow$} & IFLND: (-) LN & Chemoradiation to tumor & $\begin{array}{l}\text { Posttreatment } \\
\text { biopsy }^{b}\end{array}$ \\
\hline & & & & & $\begin{array}{l}\text { IFLND: (+) LN } \\
\text { Biopsy-proven }\end{array}$ & \multicolumn{2}{|c|}{$\begin{array}{l}\text { Chemoradiation to tumor, } \\
\text { groins, pelvis }\end{array}$} \\
\hline T2N1 & \multirow{3}{*}{$\begin{array}{l}\text { III } \\
\text { (nodal } \\
\text { spread) }\end{array}$} & IIIA & $\begin{array}{l}1 \mathrm{LN} \\
2 \mathrm{LN} \leq 5 \mathrm{~mm}\end{array}$ & \multicolumn{2}{|c|}{$\begin{array}{l}\text { Modified radical vulvectomy + groin LN } \\
\text { dissection }^{a}\end{array}$} & \multicolumn{2}{|c|}{$\begin{array}{l}\text { Chemoradiation if } \geq 2 \text { positive } \mathrm{LN} \text { or } 1 \mathrm{LN} \\
>2 \mathrm{~mm}\end{array}$} \\
\hline \multirow[t]{2}{*}{ T2N2 } & & IIIB & $\begin{array}{l}\geq 2 \mathrm{LN}>5 \mathrm{~mm} \\
\geq 3 \mathrm{LN} \leq 5 \mathrm{~mm}\end{array}$ & \multirow{3}{*}{\multicolumn{2}{|c|}{ Chemoradiation: tumor, groins, pelvis }} & & $\begin{array}{l}\text { Posttreatment } \\
\text { biopsy } \rightarrow \text { if }\end{array}$ \\
\hline & & IIIC & Extracapsular spread & & & & $(+) \rightarrow$ Surgery \\
\hline T3 & IV & IVA & $\begin{array}{l}\text { Ulcerated/fixed groin } \\
\text { LN or } \\
\text { Upper two-thirds of } \\
\text { vagina/urethra or } \\
\text { rectal/bladder mucosa }\end{array}$ & & & & $\begin{array}{l}\text { vs additional } \\
\text { chemotherapy } \pm \\
\text { radiation }\end{array}$ \\
\hline M1 & & IVB & $\begin{array}{l}\text { Distant (including } \\
\text { pelvic LN) }\end{array}$ & \multicolumn{4}{|c|}{ Chemotherapy \pm palliative radiation } \\
\hline
\end{tabular}

NOTE: Blue shading denotes surgery as a primary treatment option; orange denotes nonsurgical treatment as a primary treatment option. Abbreviations: IFLND, inguinofemoral lymphadenectomy; LN, lymph node.

${ }^{a}$ Groin lymph node dissection refers to sentinel lymph node biopsies or inguinofemoral lymphadenectomy, and is performed bilaterally if the tumor is $\geq 2 \mathrm{~cm}$ from midline.

bTo confirm complete response.

with stage IA vulvar cancer with stromal invasion of $\leq 1 \mathrm{~mm}$, treatment consists of a wide local excision without further adjuvant treatment for tumors with negative margins on final pathology. For tumors with $>1 \mathrm{~mm}$ invasion and that are up to $4 \mathrm{~cm}$ in size (stage IA, as well as small stage IB and II tumors that are $\leq 4 \mathrm{~cm}$ ), a modified radical vulvectomy is indicated, together with surgical evaluation of the groin nodes. This can be accomplished with either sentinel lymph node (SLN) biopsies, which is the preferred route for vulvar tumors $\leq 4 \mathrm{~cm}$, or IFLNDs. For a midline vulvar lesion, bilateral groin nodes rather than unilateral nodes should be evaluated, whereas for lateral lesions that are present $\geq 2 \mathrm{~cm}$ from the midline, unilateral groin nodal evaluation suffices.

Following surgical treatment, adjuvant treatment relies on margin status and additional risk factors. For tumors with positive margins, reexcision is recommended if feasible versus adjuvant external-beam RT (EBRT) to the tumor. Adjuvant RT is also considered in patients with negative margins who have other risk factors, such as the presence of lymphovascular space invasion, close (but negative) margins of $<8 \mathrm{~mm}$, large tumor size, deep invasion, and a spray or diffuse pattern of spread. Patients with nonsuspicious groin nodes who, after a modified radical vulvectomy and groin node assessment, are staged as stage IIIA (only 1 lymph node affected, or 2 lymph nodes $\leq 5 \mathrm{~mm}$ ) may also be considered for further chemoradiation if the tumor of the affected groin nodes is $>2 \mathrm{~mm}$.

\section{Locally Advanced Vulvar Cancer}

For patients with larger tumors (stage I and II with vulvar tumors $>4 \mathrm{~cm}$ ), macroscopic nodal metastases (eg, stage IIIB and IIIC), or extended localized disease to adjacent organs (stage IVA), the treatment course consists of chemoradiation, with EBRT to the tumor, groin, and pelvis, as well as concomitant chemotherapy, which usually consists of cisplatin. Primary chemoradiation is preferred for patients with 
Dellinger et al

\begin{tabular}{|lc|}
\hline $\begin{array}{c}\text { Table 2. Clinicopathologic Factors Predicting } \\
\text { Inguinal Nodal Metastases (GOG 36) }\end{array}$ \\
\hline Clinicopathologic Factors & $\begin{array}{c}\text { Risk of Inguinal Nodal } \\
\text { Metastases }\end{array}$ \\
\hline Tumor thickness & $9 \%$ \\
\hline $2 \mathrm{~mm}$ & $19 \%$ \\
\hline $3 \mathrm{~mm}$ & $31 \%$ \\
\hline $4 \mathrm{~mm}$ & $33 \%$ \\
\hline $5 \mathrm{~mm}$ & $15 \%$ \\
\hline Tumor size & $25 \%$ \\
\hline $1-2 \mathrm{~cm}$ & $36 \%$ \\
\hline $2-3 \mathrm{~cm}$ & \\
\hline $3-4 \mathrm{~cm}$ & $15 \%$ \\
\hline Grade & $20 \%$ \\
\hline 1 & $45 \%$ \\
\hline 2 & \\
\hline 3 & \\
\hline
\end{tabular}

Data from Sedlis A, Homesley H, Bundy BN, et al. Positive groin lymph nodes in superficial squamous cell vulvar cancer. A Gynecologic Oncology Group Study. Am J Obstet Gynecol 1987;156:1159-1164.

stage IB and IIB vulvar cancer with tumors $>4 \mathrm{~cm}$, with the extent of treatment dependent on the status of the groin nodes. In node-negative tumors, RT to the vulvar tumor alone may suffice, whereas in nodepositive tumors, RT should include coverage of the groin and pelvis. In clinically nonsuspicious groin nodes, surgical evaluation via an IFLND is preferred given the high false-negative rate of clinical palpation and association with lower groin recurrence rates. ${ }^{6}$ Although primary RT may have some benefit for patients unable to undergo surgery, surgical treatment of the groin remains the preferred approach. ${ }^{7,8}$ In contrast, clinically suspicious nodes may be confirmed by biopsy alone. ${ }^{5}$ For patients with stage III disease with macroscopic inguinal nodal spread (stage IIIB and IIIC) and for those with stage IVA disease, chemoradiation to the vulvar tumor, groin, and pelvis is recommended. After such treatment, assessment of disease response is recommended via tissue biopsy, and consideration should be given to additional surgery tailored toward the presence of residual disease versus additional chemotherapy. A multidisciplinary approach is necessary in most cases to individualize treatment approaches.

For patients with stage IVA vulvar cancer, surgical treatment options may include exenteration (total, anterior, or posterior pelvic) in selected candidates with isolated central disease. Neoadjuvant chemoradiation may be considered for those deemed unresectable or who prefer less radical surgical treatment. Evidence for this treatment approach exists from a phase II clinical trial, GOG $101,{ }^{9}$ in which 71 patients with T3 or T4 vulvar cancer were treated with preoperative chemoradiation, after which $48 \%$ patients had a complete response.

\section{Stage IVB}

For patients with distant metastatic disease, including pelvic lymph nodes, the treatment focus shifts to palliative chemotherapy and RT, with individualized surgical excision for palliation of pain, bleeding, and other symptoms.

\section{Surgical Treatment Options}

The surgical treatment of vulvar cancer has evolved significantly over the past century, and initially in cluded large radical and frequently disfiguring resections, which have been replaced with more conservative surgical approaches over time. The initial radical vulvectomy approach with en bloc bilateral inguinal-femoral lymphadenectomy was introduced in the sentinel papers by Taussig and Way in the 1940s. ${ }^{10,11}$ Less radical modifications were formulated by Hacker, who promoted separate groin incisions in 1981, sparing the intervening skin bridge between the groins and the vulva. ${ }^{12}$ DiSaia introduced the modified radical vulvectomy, which consisted of a wide radical excision of the primary vulvar tumor rather than resecting the entire vulva (total radical vulvectomy). ${ }^{13}$ For groin node evaluations, the requirement to perform routine groin lymphadenectomies for all vulvar cancers was modified after findings from a 1987 GOG study, ${ }^{5}$ which identified a subset of early-stage, microinvasive vulvar cancer with a negligible risk of groin nodal metastases, in whom standard groin node dissection could be omitted. Furthermore, the concept of a bilateral, full inguinofemoral lymphadenotomy was then challenged by findings from a 1993 GOG study by Homesley et al, ${ }^{14}$ in which lateralized vulvar tumors $>2 \mathrm{~cm}$ away from the midline did not metastasize to contralateral groin nodes, because they presumably drain primarily to ipsilateral groin nodes, thus resulting in the routine omission of contralateral groin node dissections for lateralized vulvar tumors and promoting unilateral groin dissections for these patients. Finally, IFLNDs have been largely replaced in smaller, 
early-stage vulvar cancers by the advent of SLN biopsies, which have been demonstrated to be feasible and reproducible in 2 prospective multicenter clinical trials. ${ }^{15,16}$ The various surgical treatment options and associated clinical evidence are outlined in the following sections.

Wide Local Excision: The terminology for a simple excision of vulvar tumor without inclusion of the deep fascia has varied significantly, and includes the following terms: wide local excision, wide deep excision, or simple vulvectomy. Such simple excision of the vulvar tumor has traditionally been reserved for preinvasive disease and stage IA vulvar cancers with $<1 \mathrm{~mm}$ stromal invasion. Generally, this procedure involves resection of the vulvar skin and a thin layer of subcutaneous fat underlying the primary vulvar tumor, with a margin of 1 to $2 \mathrm{~cm}$ circumferentially. As reported by Heaps et al, ${ }^{17}$ a lateral margin of $>8$ $\mathrm{mm}$ was associated with no recurrences, whereas those with smaller margins experienced a 50\% rate of recurrence.

Modified Radical Vulvectomy: As introduced by DiSaia et al, ${ }^{13}$ a modified radical vulvectomy includes separate incisions for the primary tumor and the groin dissections. The incision of the primary vulvar tumor is individualized based on its location, with consideration given to sparing vital organs, such as the urethra, clitoris, and anal sphincter, while ideally maintaining a 1 - to $2-\mathrm{cm}$ margin (based on the Heaps $^{17}$ study). The excision is taken down to the subcutaneous fat, superficial fascia, and deep fascia lata. Primary closure is accomplished in most cases, although reconstruction may be necessary for larger defects. Wound dehiscence and infection are common postoperative sequelae after radical vulvectomies, and patient education on wound care and perianal hygiene, as well as routine use of stool softeners and prophylactic oral antibiotics, should be considered.

Total Pelvic Exenteration: For patients with disease extending into the urethra, anus, vagina, and other organs, a pelvic exenterative approach can be a potentially curative option for well-selected patients. Several small retrospective series have reported a significant survival benefit after primary exenterative treatments for locally advanced vulvar cancers. Hopkins and Morley ${ }^{18}$ reported a series of 19 patients (11 primary, 8 recurrent vulvar cancer) who underwent posterior $(n=14)$, anterior $(n=2)$, and total $(n=3)$ pelvic exenterations, of which 10 of 14 without nodal metastases survived long-term, although none of the patients with positive lymph nodes survived; 10 of 19 had serious surgical complications. ${ }^{18}$ Similar overall survival rates were seen in a German study. ${ }^{19}$ A more recent study by MD Anderson Cancer Center included 20 pelvic exenterations for vulvar cancer, with an associated 5-year overall survival rate of $22 \%$, which was significantly worse than pelvic exenterations performed for other gynecologic cancers in this study. ${ }^{20}$ Surgical morbidity remains high and includes infectious complications, wound separation, and those complications related to urinary and gastrointestinal diversion, as well as vulvar reconstruction.

Vulvar Reconstruction: Rhomboid flaps, or myocutaneous flaps such as gracilis, gluteus, tensor fascia lata, and, more commonly, rectus abdominis, have been used; the description of these flaps have been well described elsewhere, and are beyond the scope of this review. ${ }^{21}$

Inguinofemoral Lymphadenectomy: Unilateral IFLND is recommended for patients with lateralized lesions $>2 \mathrm{~cm}$ from the midline, whereas those with medial (midline) lesions should undergo bilateral IFLND. The excision of both superficial and deep inguinal lymph nodes leads to a significantly increased risk of lymphedema and wound breakdown. Superficial inguinal lymphadenectomy alone leads to higher rates of groin recurrences $(5 \%-7 \%$ vs $1 \%$ in historical controls), as demonstrated in a GOG clinical trial. ${ }^{22}$ Several modifications to the approach of a complete IFLND have been made, including one published by Bell et $\mathrm{al}^{23}$ in 2000 , in which a complete IFLND can be performed with the preservation of the fascia lata. In this technique, an incision is made parallel to and 1 to $2 \mathrm{~cm}$ below the inguinal ligament, and taken down through Camper fascia to the lymph-containing fat below it. The femoral triangle consists of the inguinal ligament cephalad, the sartorius muscle laterally, and the adductor longus muscle medially, with the psoas muscle making up the floor of the triangle; within this triangle lie the femoral nerve, artery, vein, and lymph nodes. The saphenous vein drains into the femoral vein medially and is preserved. The medial aspect of the femoral vein is identified and often the SLN is present here. The cribriform fascia anterior to the femoral vein in the fossa ovalis is incised to remove the deep nodes en 
Dellinger et al

bloc with the superficial inguinal lymph nodes. This technique preserves the fascia lata, and retrieval of an average of 8 to 10 lymph nodes has been reported.

SLN Biopsy: Given the high risk for lower-extremity lymphedema (approximately 30\%-70\%) in patients undergoing a complete IFLND, especially when combined with RT, efforts to substitute the IFLND with less invasive procedures have been avidly researched and have culminated in the current standard of care of SLN mapping in T1 vulvar cancers. ${ }^{24}$ This method allows for surgeons to perform a limited surgery to remove the first (sentinel) lymph nodes, which receive drainage from a tumor. Pathologic information of the SLNs may subsequently triage additional decision-making for a more extensive lymphadenectomy or adjuvant RT.

Two prospective multicenter studies, GOG 173 and GROINSS-V-1, have demonstrated the safety and feasibility of SLN mapping of the groin in vulvar cancer. ${ }^{15,16}$ GOG 173 was a prospective study in early-stage vulvar cancer $(<6 \mathrm{~cm})$, in which patients underwent a radical vulvectomy along with SLN mapping, followed by standard complete IFLND. In GROINSS-V-1, patients with early $\mathrm{T} 1$ and $\mathrm{T} 2$ tumors $<4 \mathrm{~cm}$ were enrolled and subjected to SLN mapping, followed by complete IFLND if the SLN biopsy was positive by pathology or ultrastaging. The false-negative rate of an SLN biopsy in GOG 173 was $2.7 \%$ in patients whose tumors were $<4 \mathrm{~cm}$. In contrast, the GROINSS-V-1 study reported an isolated groin recurrence rate of $2 \%$. These results were considered safe for incorporation into standard practice for patients with early-stage vulvar cancers with unifocal tumors $<4 \mathrm{~cm}$ in size who do not have evidence of suspicious groin adenopathy. It is important to note that recurrence in the groin is usually a fatal event, and thus a critical outcome measure in this disease. A recent systematic review and metaanalysis of the cumulative data on SLN detection reported a per-groin detection rate of $87 \%$ and a false-negative rate of $6.4 \%$. The reported recurrence rates among complete versus superficial IFLND versus SLN biopsy were $1.4 \%, 6.6 \%$, and 3.4\%, respectively. ${ }^{24}$ The current standard of care for the detection of the inguinal SLN includes the dual use of lymphoscintigraphy with technetium $99 \mathrm{~m}\left({ }^{99 \mathrm{~m}} \mathrm{Tc}\right)$, in combination with an intraoperative injection of blue dye (methylene blue or indigo carmine) (Figure 1). In GOG 173, the false-negative rates were improved by the combination of ${ }^{99 \mathrm{~m}} \mathrm{Tc}$ and blue dye

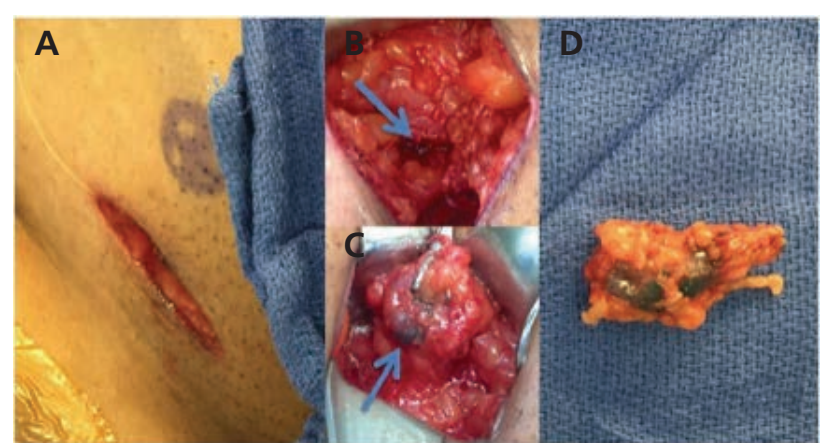

Figure 1. Sentinel lymph node mapping and biopsy. (A) A 4-cm incision is made parallel to the inguinal ligament, and the subcutaneous fat is incised. (B, C) A blue node is visualized (arrows), confirmed to be at least 10 -fold more radioactive than baseline on gamma probing, and (D) excised.

(1.6\%), as opposed to blue dye alone $(2.0 \%)$ or ${ }^{99 \mathrm{~m}} \mathrm{Tc}$ alone $(7.8 \%) .{ }^{15}$ In contrast, a recent meta-analysis found no significant difference in detection rate per groin between the combination of blue dye and ${ }^{99 \mathrm{~m}} \mathrm{Tc}$ (87\%) compared with ${ }^{99 \mathrm{~m}} \mathrm{Tc}$ alone $(84 \%)$, although the detection rate was higher with the combination than with dye alone (63\%). ${ }^{24}$ The dual use of ${ }^{99 \mathrm{~m}} \mathrm{Tc}$ with or without blue dye is recommended for the detection of SLNs, whereas the use of blue dye alone is discouraged due to a low detection rate.

The most common site for the SLN is the medial aspect of the femoral vein, with a second echelon LN found frequently just above the inguinal ligament. Inability to map should be followed by a complete IFLND. Postoperative pathologic diagnosis with or without ultrastaging directs further treatment, including completion IFLND versus adjuvant RT therapy. The decision to proceed to either IFLND or RT is the subject of an ongoing prospective trial (GOG 270/GROINSS-V-II), which is prospectively evaluating the safety of adjuvant groin RT in lieu of full IFLND in women with micrometastases detected in SLNs. Ultrastaging describes a more detailed pathologic examination, using a combination of hematoxylin and eosin staining with cytokeratin on paraffin-embedded SLN tissue cut at smaller intervals to determine presence of micrometastases. Although the detection of micrometastases in SLNs via ultrastaging has led to improved detection of positive groin nodes ( $41 \%$ of positive SLNs in the GROINSS-V study were detected by ultrastaging), the value of such findings remains debated, because the overall survival of patients with positive SLNs detected via ultrastaging remained relatively good compared with 
Vulvar Cancer Treatment

that of patients with positive SLNs detected by routine microscopy ( $89 \%$ vs $65 \% ; P=.002$ ).

\section{Discussion}

Surgical treatment of vulvar cancer has evolved over the past several decades, from a radical en bloc resection to a more individualized, conservative surgical approach, including wide local or wide radical excisions for microinvasive and early-stage disease, respectively, as well as separate groin incisions, with more conservative groin dissections to reduce quality of life issues, such as lymphedema, sexual dysfunction, and urinary and gastrointestinal sequelae. Pelvic exenteration is reserved for well-selected patients with local advanced-stage vulvar cancers, and consideration should be given to neoadjuvant chemoradiation to reduce short-term and longterm morbidity. The introduction of SLN biopsies has largely replaced IFLNDs in early-stage vulvar cancers, although more studies are needed to further delineate the application and outcomes of such approach. These questions include the optimal postoperative management of positive SLNs, because current guidelines equally advocate return to the operating room for a complete IFLND versus proceeding directly to adjuvant RT. The results of the ongoing GROINSS-V-II/GOG study are eagerly awaited to answer this question. Equally uncertain is the role of ultrastaging of SLNs and the significance on prognosis and adjuvant treatment; the true value of SLN ultrastaging may not be known until lymph nodes obtained from traditional IFLND are also subjected to ultrastaging. ${ }^{25}$ Lastly, more emphasis has been placed in recent years on quality-of-life issues, and specifically the question of lymphedema development in patients with vulvar cancer. Recent data suggest that the incidence of lower extremity lymphedema has been greatly underestimated, with rates as high as $60 \%$ to $67 \%$ at 6 months postoperatively after IFLND, based on a prospective interventional trial conducted by the GOG. ${ }^{26}$ In contrast, a large multicenter observational study on SLN detection reported lower extremity lymphedema rates of $25 \%$ after IFLND versus $2 \%$ after SLN biopsy. ${ }^{27}$ The varying incidence rates and lack of effective preventive treatments underscore the need for further study in this field, such as the recently completed cooperative trial (GOG 244), an observational multicenter study that prospectively evaluated the incidence of lymphedema after surgery for vulvar and other gynecologic cancers.

\section{References}

1. Siegel RL, Miller KD, Jemal A. Cancer statistics, 2016. CA Cancer J Clin 2016;66:7-30.

2. Modesitt SC, Waters AB, Walton $\mathrm{L}$, et al. Vulvar intraepithelial neoplasia III: occult cancer and the impact of margin status on recurrence. Obstet Gynecol 1998;92:962-966.

3. Greer BE, Koh WJ. New NCCN Guidelines for Vulvar Cancer. J Natl Compr Canc Netw 2016;14:656-658.

4. Kamran MW, O'Toole F, Meghen K, et al. Whole-body [18F]fluoro-2deoxyglucose positron emission tomography scan as combined PET CT staging prior to planned radical vulvectomy and inguinofemoral lymphadenectomy for squamous vulvar cancer: a correlation with groin node metastasis. Eur J Gynaecol Oncol 2014;35:230-235.

5. Sedlis A, Homesley H, Bundy BN, et al. Positive groin lymph nodes in superficial squamous cell vulvar cancer. A Gynecologic Oncology Group Study. Am J Obstet Gynecol 1987;156:1159-1164.

6. Stehman FB, Bundy BN, Thomas G, et al. Groin dissection versus groin radiation in carcinoma of the vulva: a Gynecologic Oncology Group study. Int J Radiat Oncol Biol Phys 1992;24:389-396.

7. Hallak S, Ladi L, Sorbe B. Prophylactic inguinal-femoral irradiation as an alternative to primary lymphadenectomy in treatment of vulvar carcinoma. Int J Oncol 2007;31:1077-1085

8. Petereit DG, Mehta MP, Buchler DA, Kinsella TJ. Inguinofemoral radiation of NO,N1 vulvar cancer may be equivalent to lymphadenectomy if proper radiation technique is used. Int J Radiat Oncol Biol Phys 1993;27:963-967.

9. Moore DH, Thomas GM, Montana GS, et al. Preoperative chemoradiation for advanced vulvar cancer: a phase II study of the Gynecologic Oncology Group. Int J Radiat Oncol Biol Phys 1998;42:79-85.

10. Taussig FJ. Cancer of the vulva: an analysis of 155 cases (1911-1940) Am J Obstet Gynecol 1949;40:764-772.

11. Way S. Carcinoma of the vulva. Am J Obstet Gynecol 1960;79:692697.

12. Hacker NF, Leuchter RS, Berek JS, et al. Radical vulvectomy and bilateral inguinal lymphadenectomy through separate groin incisions. Obstet Gynecol 1981;58:574-579.

13. DiSaia PJ, Creasman WT, Rich WM. An alternate approach to early cancer of the vulva. Am J Obstet Gynecol 1979;133:825-832.

14. Homesley HD, Bundy BN, Sedlis A, et al. Prognostic factors for groin node metastasis in squamous cell carcinoma of the vulva (a Gynecologic Oncology Group study). Gynecol Oncol 1993;49:279-283.

15. Levenback CF, Ali S, Coleman RL, et al. Lymphatic mapping and sentinel lymph node biopsy in women with squamous cell carcinoma of the vulva: a Gynecologic Oncology Group study. J Clin Oncol 2012;30:3786-3791

16. Oonk $\mathrm{MH}$, van Hemel BM, Hollema $\mathrm{H}$, et al. Size of sentinel-node metastasis and chances of non-sentinel-node involvement and survival in early stage vulvar cancer: results from GROINSS-V, a multicentre observational study. Lancet Oncol 2010;11:646-652.

17. Heaps JM, Fu YS, Montz FJ, et al. Surgical-pathologic variables predictive of local recurrence in squamous cell carcinoma of the vulva. Gynecol Oncol 1990;38:309-314.

18. Hopkins MP, Morley GW. Pelvic exenteration for the treatment of vulvar cancer. Cancer 1992;70:2835-2838.

19. Forner DM, Lampe B. Exenteration in the treatment of stage III/IV vulvar cancer. Gynecol Oncol 2012;124:87-91.

20. Westin SN, Rallapalli V, Fellman B, et al. Overall survival after pelvic exenteration for gynecologic malignancy. Gynecol Oncol 2014;134:546-551.

21. Hockel M, Dornhofer N. Vulvovaginal reconstruction for neoplastic disease. Lancet Oncol 2008;9:559-568.

22. Stehman FB, Bundy BN, Ball H, Clarke-Pearson DL. Sites of failure and times to failure in carcinoma of the vulva treated conservatively: a Gynecologic Oncology Group study. Am J Obstet Gynecol 1996;174:1128-1132; discussion 1132-1133.

23. Bell JG, Lea JS, Reid GC. Complete groin lymphadenectomy with preservation of the fascia lata in the treatment of vulvar carcinoma. Gynecol Oncol 2000;77:314-318. 
24. Covens A, Vella ET, Kennedy EB, et al. Sentinel lymph node biopsy in vulvar cancer: systematic review, meta-analysis and guideline recommendations. Gynecol Oncol 2015;137:351-361.

25. Slomovitz BM, Coleman RL, Oonk MH, et al. Update on sentinel lymph node biopsy for early-stage vulvar cancer. Gynecol Oncol 2015;138:472-477.
26. Carlson JW, Kauderer J, Walker JL, et al. A randomized phase III trial of $\mathrm{VH}$ fibrin sealant to reduce lymphedema after inguinal lymph node dissection: a Gynecologic Oncology Group study. Gynecol Oncol 2008;110:76-82.

27. Van der Zee AG, Oonk MH, De Hullu JA, et al. Sentinel node dissection is safe in the treatment of early-stage vulvar cancer. J Clin Oncol 2008;26:884-889.

\section{Instructions for Completion}

To participate in this journal CE activity: 1) review the learning objectives and author disclosures; 2) study the education content; 3) take the posttest with a $66 \%$ minimum passing score and complete the evaluation at http://education.nccn.org/ node/80141; and 4) view/print certificate. After reading the article, you should be able to answer the following multiple-

\section{Posttest Questions}

1. True or False: Standard surgical management of early-stage vulvar cancer has evolved to include less radical resection of the primary tumor and separate incisions for inguinofemoral lymph node evaluation.

2. Decisions regarding adjuvant RT following complete resection of early-stage vulvar cancer (negative margins) should take into account the following risk factors:
a. Lymphovascular space invasion
b. Close margins $(<8 \mathrm{~mm})$
c. Large tumor size
d. Depth of invasion

choice questions. Credit cannot be obtained for tests completed on paper. You must be a registered user on NCCN.org. If you are not registered on NCCN.org, click on "New Member? Sign up here" link on the left hand side of the Web site to register. Only one answer is correct for each question. Once you successfully answer all posttest questions you will be able to view and/or print your certificate. Software requirements: Internet e. Spray or diffuse pattern of disease

f. All of the above

3. Potential treatment options for stage III/IV vulvar cancer include which of the following procedures?

a. Primary chemoradiation

b. Wide local excision

c. SLN mapping

d. Pelvic exenteration

e. A and C

f. A and D

g. B and C

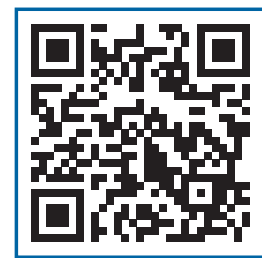

